\title{
DNA overstretching transition induced by melting in a dynamical mesoscopic model
}

\author{
Ana Elisa Bergues Pupo, ${ }^{1,2}$ Fernando Falo, ${ }^{1,3}$ and Alessandro Fiasconaro ${ }^{1,4, a)}$ \\ ${ }^{1}$ Departamento de Física de la Materia Condensada, Universidad de Zaragoza, 50009 Zaragoza, Spain \\ ${ }^{2}$ Departamento de Física, Universidad de Oriente, 90500 Santiago de Cuba, Cuba \\ ${ }^{3}$ Instituto de Biocomputación y Física de Sistemas Complejos (BIFI), Universidad de Zaragoza, \\ 50009 Zaragoza, Spain \\ ${ }^{4}$ Instituto de Ciencia de Materiales de Aragón, CSIC-Universidad de Zaragoza, 50009 Zaragoza, Spain
}

(Received 3 May 2013; accepted 12 August 2013; published online 3 September 2013)

\begin{abstract}
We present a phenomenological dynamical model describing the force induced melting as responsible for the DNA overstretching transition. The denaturation mechanism is developed under the framework of the mesoscopic one-dimensional Peyrard-Bishop-Dauxois (PBD) picture which models the melting features of a polymer chain by means of a Morse potential and the stacking interaction. We find a good agreement with both the experimental overstretching curve and the asymmetric hysteretic properties with different simulation times. The comparison of the standard PBD model with a modification of the Morse potential which takes into account the interaction with the solvent has been also successfully investigated. @ 2013 AIP Publishing LLC. [http://dx.doi.org/10.1063/1.4819263]
\end{abstract}

\section{INTRODUCTION}

The development of the experimental techniques at the nanoscale and even the possibility of visualizing and manipulating single molecules have given a big impulse to the study and characterization of the mechanical properties of biological object. ${ }^{1}$

One of the most intriguing and only partially clear phenomena is the so-called overstretching transition of the DNA, ${ }^{2,3}$ which consists in a sudden elongation of the DNA chain of about $70 \%$ more than the native length, when a force around $70 \mathrm{pN}$ (the overstretching force) is applied. The presence of this tension-induced overstretching transition reveals the existence of two structurally different DNA states: the BDNA, at low forces, where the base pairs are packed and helicoidally twisted in their native state, and another state, present at high forces, that was first called the $\mathrm{S}$ state, where the base pair (bp) distance is higher by about $70 \%$ than that in the B state.

After more than a decade, the nature of this transition, as well as the molecular structure of the overstretched state, remain controversial. Three main mechanisms could be involved in this transition. Two of them involve strand separation: peeling from nicks in the chain or its free edge, where one strand retracts from its complementary strand via basepair breaking; and inside-strand separation, where the two complementary tracts of the double stranded DNA (dsDNA) both remain under tension, but localized openings of denaturation are created throughout the molecule. ${ }^{4-6}$ This idea was supported by the dependence of the overstretching force on parameters that also affects the melting temperature such as $\mathrm{pH}$ and salt concentration. In the stretching dynamics, it has been observed the presence of an asymmetric hysteresis in the elongation curve when the force increases or decreases, which

\footnotetext{
a)Electronic mail: afiascon@unizar.es
}

depends both on temperature and pulling rates. The presence of this hysteresis, due to the slow recombination kinetics of the strands in the decreasing of the pull force, would support the idea of a force induced denaturation of the DNA (melting), which gives rise to the so-called M-DNA.

In the other mechanism, the two strands remain connected each other, i.e., the base pairs remain bonded, and the overstretching appears from a cooperative chain unwinding. ${ }^{2,3,7-9}$ This state is the so-called S-DNA and its justification appears from the geometrical and the mechanical properties of DNA.

Recent experiments would show that all these mechanisms can be superimposed depending on DNA topology and experimental conditions. ${ }^{10-16}$ S-DNA is favored at low temperatures, high salt concentration, and high Cytosine-Guanine (CG) pairs content, while, on the contrary, strand separation is possible at conditions that affect base pair stability. At standard temperatures and solution concentration both mechanisms can compete.

As a support of the experiments, several simulations reproducing the overstretching transition have been proposed: statistical-mechanics models, which mainly follow the WLC model, ${ }^{7,8,17}$ and take into account stretch-twist coupling. ${ }^{12,18}$ Full-atom molecular dynamic simulations ${ }^{19}$ or mesoscopic models $\mathrm{s}^{20-24}$ have been implemented in order to depict the different phenomenologies.

The standard melting transition of DNA with the temperature has been widely studied both theoretically and experimentally. One of the most studied models for it is the PeyrardBishop-Dauxois (PBD) mesoscopic model. ${ }^{25}$ It describes the stretching of the bonds between the bps through a single variable, which condenses all the atomic coordinates of a bp. It describes quite well the relative separation of two bases of double stranded DNA when temperature is increased.

Besides the standard form of the model, which makes use of a Morse potential between the base pairs, a recent 
modification of the potential with a return barrier have also included the interaction with the solvent with the result of giving the width of the melting transition, as well as the size of the thermal bubbles of DNA, closer to the experimental results. $^{26,27}$

PBD model has been also successfully used in describing some mechanical properties of DNA, like mechanical unzipping, ${ }^{28,29}$ i.e., the separation of the double strand by pulling one strand far away from the other, and also with the presence of the barrier and the variation of the potential deepness with the salt concentration in the solution. ${ }^{30}$

Taking into account the similarities between melting processes assisted by temperature and force, we propose a dynamic and mesoscopic model able to reproduce the overstretching features focusing on the hypothesis that the overstretching is essentially due a force induced melting. This model is a step forward to a previous investigation ${ }^{22}$ where the overstretching transition has been dynamically obtained in a pure phenomenological approach by means of a double well potential without reference to any inner mechanism of the overstretching process. A comparison with the two different versions of the PBD model has been also studied here.

\section{MODEL AND METHODS}

In accordance with a previous model, ${ }^{22}$ the DNA is modelled here as a three-dimensional chain of $\mathrm{N}$ monomers connected by springs. We do not need to investigate again the results for low and intermediate forces already studied there, we focus here around the overstretching transition, which occurs when the chain is almost fully stretched. For this reason it is not necessary to include the terms of bending and LennardJones energy in principle present in our description, ${ }^{22}$ but not influent at that range of forces. In the present model, the potential of consecutive monomers is considered as harmonic, in formula $U=\frac{1}{2} k_{U}\left(l_{i}-l_{0}\right)^{2}$, where $l_{i}=\left|\mathbf{r}_{i+1}-\mathbf{r}_{i}\right|$ is the separation between the two monomers, with $\mathbf{r}_{i}$ s their vector position in the space, and $l_{0}$ is the equilibrium separation between them.

The overdamped equation of motion is then

$$
\dot{\mathbf{r}}_{i}=-\nabla_{i} U\left(\mathbf{r}_{i}\right)+F\left(\delta_{i, n}-\delta_{i, 1}\right) \overrightarrow{\mathbf{i}}+\sqrt{2 k_{B} T} \vec{\xi}_{i}(t),
$$

where $\vec{\xi}_{i}(t)=\left(\xi_{i}^{x}(t), \xi_{i}^{y}(t), \xi_{i}^{z}(t)\right)$ represents the thermal contribution as a Gaussian uncorrelated noise $\left(\left\langle\xi_{i}^{\alpha}(t) \xi_{j}^{\beta}\left(t^{\prime}\right)\right\rangle\right.$ $\left.=\delta_{i, j} \delta_{\alpha, \beta} \delta\left(t-t^{\prime}\right)\right)$ with zero mean $\left(\left\langle\xi_{i}^{\alpha}(t)\right\rangle=0\right)$, and the time $t$ is scaled with the damping $\gamma$ as $t \rightarrow t / \gamma$. Two forces $F$ and $-F$ act on the chain in the $x$ direction on the first and last monomer, respectively, in order to stretch the polymer. We assume that when the polymer is stretched beyond a value of force, a separation of some regions of the double strand can occur. This latter describes the force induced melting process. The separation of the chain at monomer $i$ is introduced by means of the dynamical variable $d_{i}$, representing the difference in the separation of the chain with respect the equilibrium value of the relaxed chain. When the value of the variable $d_{i}$ exceeds a threshold, the chain undergoes a melting transition in that region, and the separation between adjacent monomers increases (see also Fig. 1). (a)

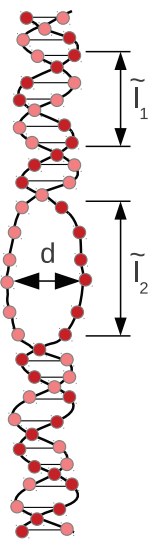

(b)

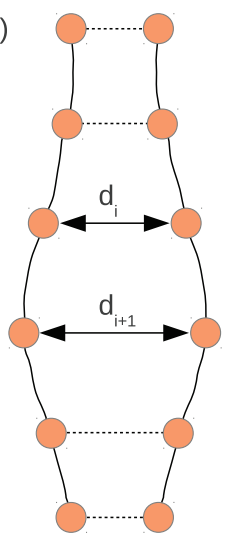

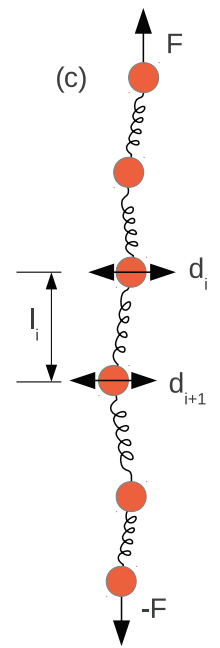

FIG. 1. Schematic model representation. (a) dsDNA structure with some bps in the B-DNA state (equilibrium separation $\tilde{l}_{1}$ ) and others in the M-DNA state (equilibrium separation $\tilde{l}_{2}$ ). (b) Strand separation at each monomer site. Each monomer represents several bps. (c) Linear chain and degrees of freedom of each monomer: $l_{i}$, the distance between two consecutive monomers and $d_{i}$, the state of opening at each monomer site.

In order to relate the melting and elongation, the equilibrium separation between monomers has been modeled as dependent on the melting state of the two neighbors, i.e., $l_{0}=$ $l_{0}\left(d_{i}, d_{i+1}\right)$, with the following dependence: if $d_{i}<\tilde{d}_{1}, l_{0}$ is the equilibrium length $\tilde{l}_{1}$ (corresponding to B-DNA state), while for $d_{i}>\tilde{d}_{2}$ the length is $\tilde{l}_{2}$ (corresponding to overstretched DNA). We use in the interval between the two thresholds a linear dependence

$$
l_{0}\left(d_{i}, d_{i+1}\right)=\tilde{l}_{1}+\frac{1}{2} \frac{\tilde{l}_{2}-\tilde{l}_{1}}{\tilde{d}_{2}-\tilde{d}_{1}}\left[\left(d_{i+1}-\tilde{d}_{1}\right)+\left(d_{i}-\tilde{d}_{1}\right)\right]
$$

The term so written takes into account that the length of the $i$ th spring, depends on the contribution of the opening state of two consecutive monomers: the $i$ th and the $(i+1)$ th.

As concern the already presented melting distance $d_{i}$, we modelled its behavior, in the context of the PBD model, as a dynamic variable subject to an independent stochastic equation,

$$
\dot{d}_{i}=-\nabla_{i} V\left(d_{i}\right)-\nabla_{i} W\left(d_{i}, d_{i-1}\right)+\sqrt{2 k_{B} T} \zeta_{i}(t),
$$

with the noise satisfying the usual properties $\left\langle\zeta_{i}(t)\right\rangle=0$, and $\left.\left\langle\zeta_{i}(t)\right\rangle \zeta_{j}\left(t^{\prime}\right)\right\rangle=\delta_{i, j} \delta\left(t-t^{\prime}\right)$. The potential bonding energy of the nucleotides of every base is given by both the Morse potential $V\left(d_{\mathrm{i}}\right)$ and the staking interaction $W\left(d_{i}, d_{i-1}\right)$. We use here not only the standard form for the Morse potential $V$, but also a modified version recently used in Ref. 26, in the form applied in Ref. 27 in order of sharpening the melting transition with the temperature. In this latter version, a Gaussian barrier is added for describing the interaction between the two nucleotides with the solvent once the base is already open. In this case the contribution of the solvent makes the recombination of the two nucleotides more difficult, and the potential presents a barrier in the closure path of the nucleotides, i.e., for decreasing values of the variable $d_{i}$. The two versions of 
the potential are shown in Fig. 2, and the analytic expression is

$$
V\left(d_{i}\right)=D\left(\mathrm{e}^{-\alpha d_{i}}-1\right)^{2}+G \mathrm{e}^{-\left(d_{i}-y_{0}\right)^{2} / b} .
$$

The first term in the sum is the usual form of the Morse potential, where the $D$ is the depth of the potential which represents the energy needed to dissociate the strands at each monomer site, and $\alpha$ is the width of the well. The second term in the sum is the Gaussian barrier, whose height, width, and position are given by $G, b$, and $y_{0}$, respectively. The standard form of the potential is recovered by setting $G=0$.

The stacking interaction has the shape

$$
W\left(d_{i}, d_{i-1}\right)=\frac{1}{2} k_{W}\left(1+\rho e^{-\delta\left(d_{i}+d_{i-1}\right)}\right)\left(d_{i}-d_{i-1}\right)^{2} .
$$

The effect of the exponential term, whose intensity is governed by $\rho$, is to change the effective coupling constant from $k_{W}(1+\rho)$ to $k_{W}$ when one of the bps is displaced far away from its equilibrium position. The parameter $\delta$ in the exponential function sets the scale length of this action. This potential takes into account the recoil force contribution given by the adjacent nucleotides in the single DNA strand once the base is separated in the melting process. For simplicity we will consider first the stacking interaction to be harmonic, i.e., $\rho=0$. The effect of anharmonicity will be studied at the end of the paper.

The melting transition is a cooperative process where the bases open when increasing the temperature. If $k_{B} T / D \ll 1$, the bases remain closed. As this ratio approaches 1, bases separation increases and the melting of the chain occurs. However, stretching experiments on DNA are conducted at constant temperature and below melting transition temperature (which occurs at zero applied forces). For this reason, with the purpose to induce melting in the system, we infer that $D$ decreases when increasing the stretching force. Also considering the not completely abrupt behavior of the OS transition, we suppose for $D$ the sigmoidal dependence shown in the inset of Fig. 2:

$$
D=D(F)=D_{0}-\frac{h}{1+e^{-p\left(F-F_{o s}\right)}},
$$

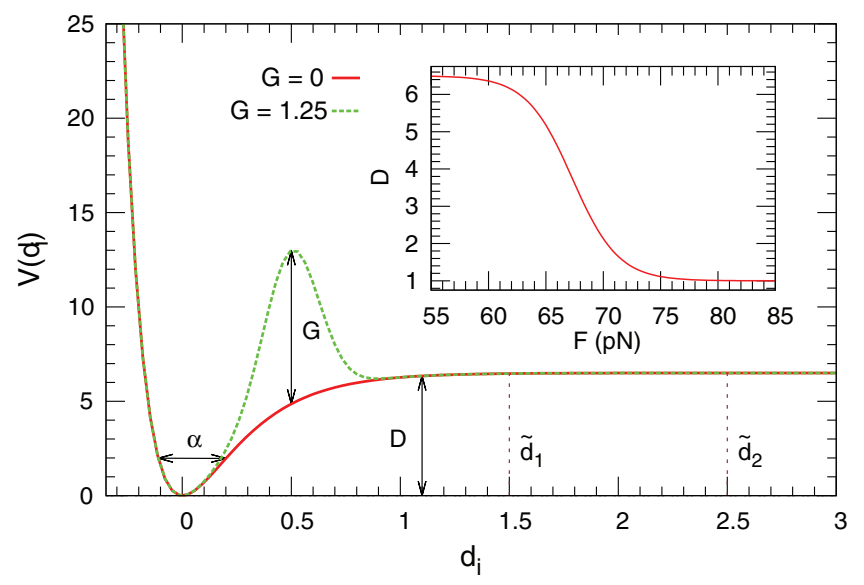

FIG. 2. Morse potential without barrier $(G=0)$ and with barrier $(G=1.25)$. $\tilde{d}_{1}$ and $\tilde{d}_{2}$ are the threshold values for the change of $l_{0}$ with $d$. (Inset) Dependence of parameter $D$ with the applied force $F$. where $F_{o s}$ is the overstretching force, $D_{0}$ is the value of the depth of the Morse potential at zero applied forces, and $h$ and $p$ are two parameters indicating the depth and the width of the variation of $D$, respectively. The presence of this phenomenological term allows the force induced melting occurring at large forces, leaving the low ones practically unperturbed.

The model units are the same than in Ref. 22. We set the units of energy and length to be $E_{u}=4.1 \mathrm{pN} \mathrm{nm}$ (thermal energy at room temperature) and $l_{u}=5.3 \mathrm{~nm}$, respectively. With these units the parameters used in the simulations are $k_{B} T=E_{u}, k_{U}=1500 E_{u} / l_{u}^{2}, \tilde{l}_{1}=l_{u}, \tilde{l}_{2}=1.67 l_{u}$. For the PBD model we use $\alpha=4 l_{u}^{-1}$ and $k_{W}=0.14 E_{u} / l_{u}^{2}$. Following Ref. 27, we set $y_{0}=2 / \alpha$ and $b=1 / 2 \alpha^{2}$. Parameters for the dependence of $D$ on $F$ are: $D_{0}=6.5 E_{u}, h=5.5 E_{u}$, and $p=0.5 l_{u} / E_{u}$.

If we compare the magnitude of our PBD parameter values with those used for melting transition in Ref. 25, we note some differences, due to the fact that the original PBD model has been proposed to describe interactions between nucleotides of a single bp, while here a single monomer is compound by several bps. As a consequence, the dissociation energy $D$ is larger, because it represents the energy needed to separate several nucleotides, and, at the same way, the stacking interaction can be regarded as springs connecting consecutive bps. In this case the coupling constant $k_{W}$ for consecutive monomers is the equivalent of several serially connected springs, and its value is lower than that one of each individual spring (see also Ref. 31).

\section{RESULTS}

\section{A. Morse potential $(G=0)$}

We explore the shape of the stretching curves using Eq. (3) without barrier, i.e., $G=0$ with an overstretching force $F_{o s}=67.3 \mathrm{pN}$. In the experiments the ends of the chain are maintained fixed to some device. For this reason we set in all simulations $d_{1}=0$ and $d_{N}=0$. In order to make sure that equilibrium conditions are reached we chose a simulation time such that the forward and backward curves coincide. Thus, a time of $15 \times 10^{6}$ steps is used for equilibrium simulations. Simulations are then averaged over 10 different realizations of the trajectories. The computer simulations have been done with a Runge-Kutta stochastic algorithm using $d t=0.01$.

The main plot of Fig. 3 shows the length of the stretched DNA with respect its contour length $\left(L_{x} / L_{c}\right)$ as a function of the applied force $F$. A good correspondence between the simulation and experimental data is visible. The right inset shows the surface plot of the mean distance between the two strands of the DNA chain $\left(\left\langle d_{i}\right\rangle\right)$, as a function of both the index of the monomer $i$, and the applied force $F$. The value $\left\langle d_{i}\right\rangle$ is defined as the time average of the separation between monomer during the simulation, averaged once more on 10 different realizations $\left\langle d_{i}\right\rangle=\frac{1}{10} \frac{1}{t_{s}} \sum_{j=1}^{10} \int_{t_{s}} d_{j}(t) d t$. The homogeneity of the color level along the various monomers indicates that a good saturation values is reached in the simulations. The same information is evident from the left inset, where $\left\langle d_{i}\right\rangle$ is shown for three applied forces (before, during, and after the 


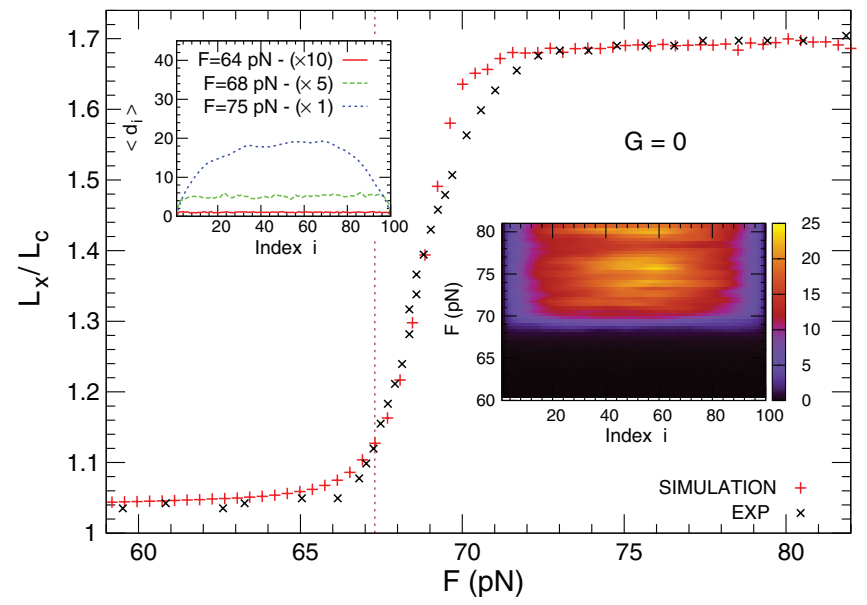

FIG. 3. DNA length vs applied force $F$ with the Morse potential without barrier $(G=0)$. (Right inset) Mean displacement $\left\langle d_{i}\right\rangle$ of every monomer ( $x$-axis), and for the different applied forces ( $y$-axis). (Left inset) A section of $\left\langle d_{i}\right\rangle$ as a function of the monomer index $i$ for three selected values of the force. The values of $\left\langle d_{i}\right\rangle$ for the forces $F=64 \mathrm{pN}$ and $F=68 \mathrm{pN}$ have been scaled with a factor $\times 10$, and 5 , respectively.

transition) showing a reasonable smooth distribution between the distances. It is worth to note that the melting here simulated is not total, as the two edges of the chain are maintained at the equilibrium distances, this implies that the two strand cannot separate undefinitely, and an equilibrium configuration of the chain is thus reached. The probability distribution for the distance between two adjacent monomers $l_{i}$ and for the melting variable $d_{i}$ have been also evaluated for different values of the applied force (see Fig. 4).

One important feature that has been commonly associated to the melting mechanisms involved in the strand separation is the existence of a clear hysteresis in the DNA elongation curve if measured when the chain is stretched and the force $F$ is progressively increased, or, on the contrary, when the force is progressively decreased. The two curves built that way can show different hysteretic paths. ${ }^{4,5,32,33}$ However, in a recent experiment ${ }^{15}$ it has been shown that hysteretic behavior is present when strand separation is due to unpeeling while non-hysteretic is obtained for a mere melting

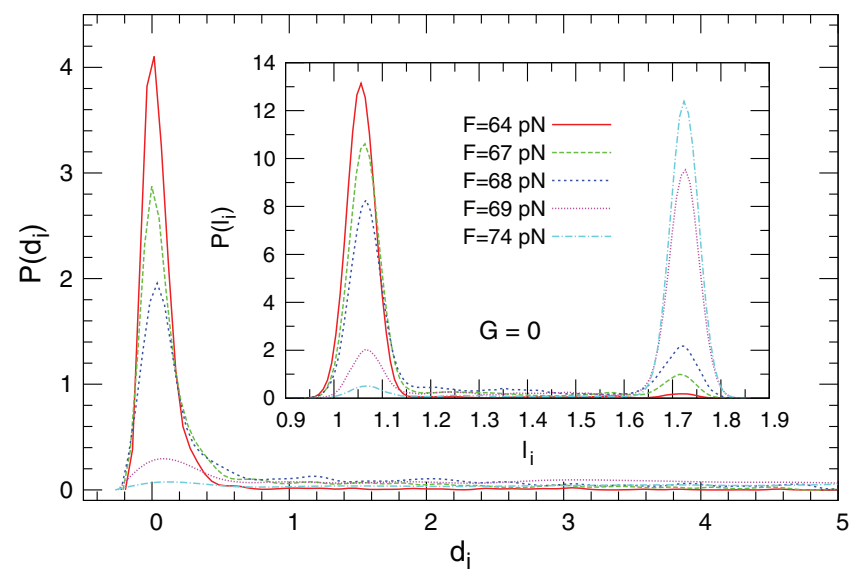

FIG. 4. Probability distribution of the monomer distance $l_{i}$ and of the melting variable $d_{i}$ for different values of the applied force $F$.
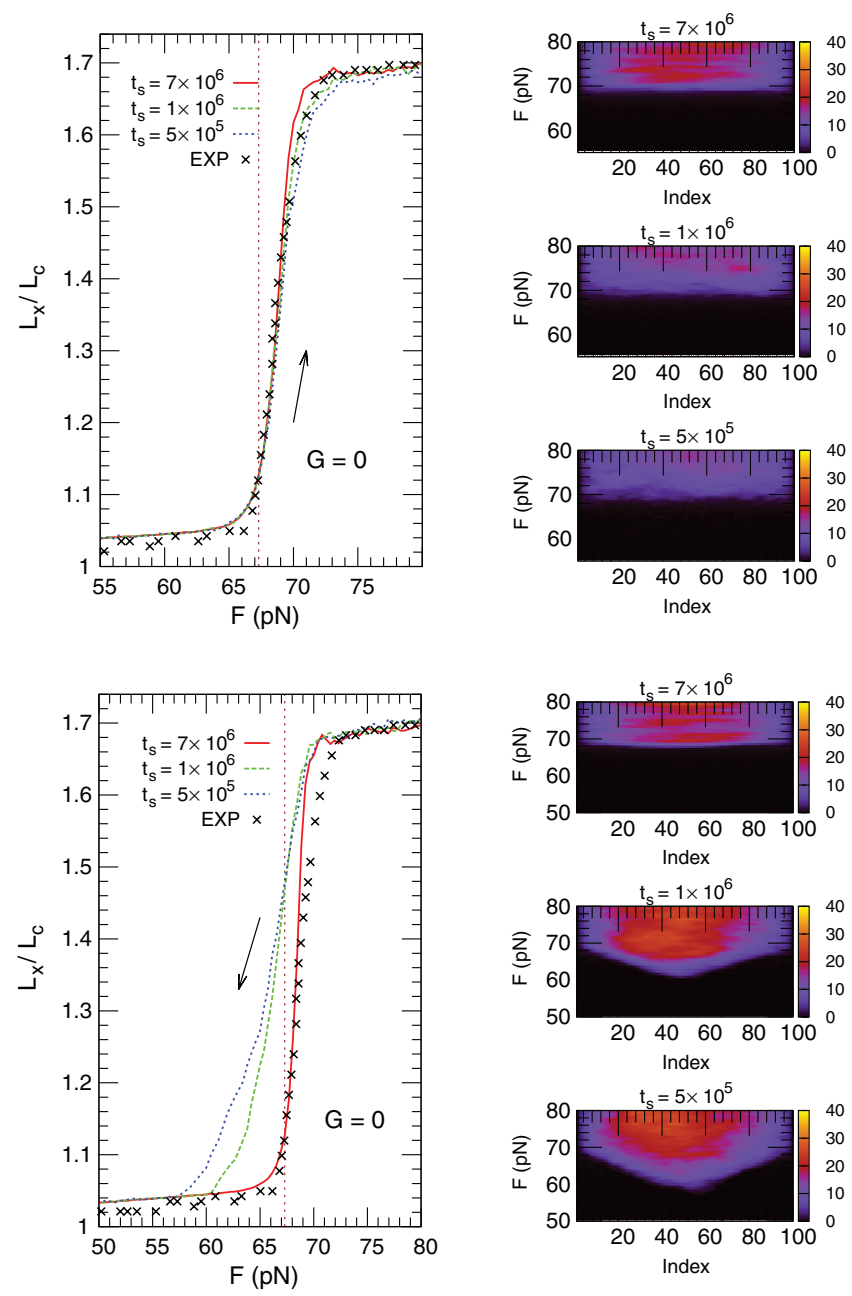

Index

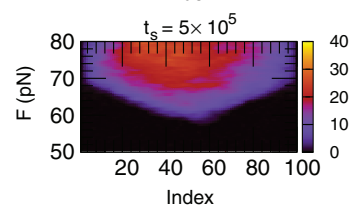

FIG. 5. Transition with different simulation times. Upper panels: Forward direction (pulling). Lower panels: Backward direction (releasing).

mechanism. One possible explanation of the presence of hysteresis without rupture of the strands can be due to the relaxation time of the system. ${ }^{7,22}$ The idea that the recombination of the two strands after the melting can give rise to asymmetric hysteresis can be reasonable by considering the different times necessary for the chain getting molten with respect to that one taken by its recombination when decreasing the force. In fact, hysteresis can appear when no enough time for reaching the equilibrium is used, and in this sense numerical experiments can easily reproduce the experimental outcomes, taking also into account that the time necessary for reaching the equilibrium can depend on the specific kind of mechanism.

For the simulation time used previously, no hysteresis is observed when the equilibrium is successfully reached. When decreasing the simulation time $t_{s}$, which is equivalent to increase the pulling rate in both forward and backward experiment, the curves shown in Fig. 5 are obtained. Upper insets report the pulling for increasing forces and the lowers for decreasing forces, respectively, for different values of $t_{s}$. Differently from the previous symmetrical model, ${ }^{22}$ the observed hysteresis is here asymmetrical, due to the implicit asymmetry of the Morse potential describing the melting features of 


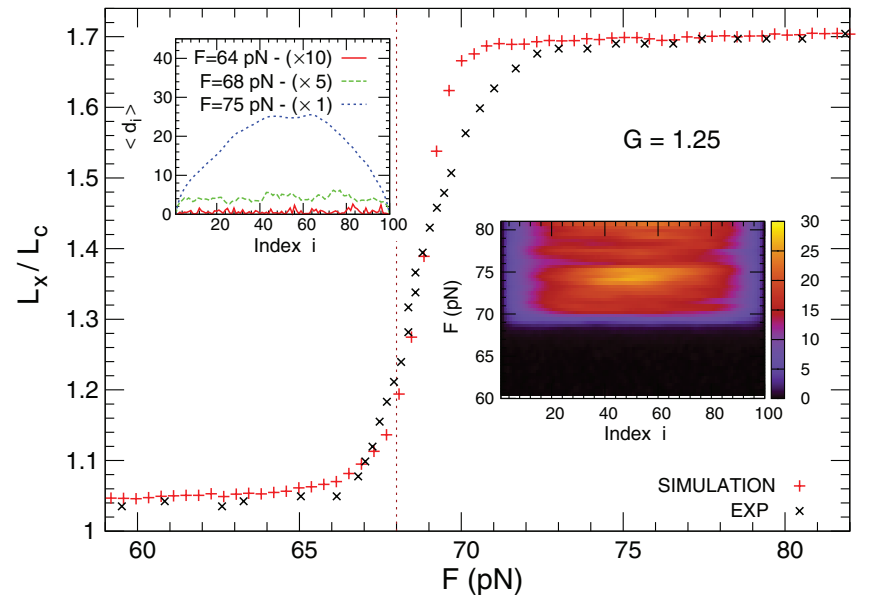

FIG. 6. DNA length vs applied force $F$ with the Morse potential with barrier $(G=1.25)$. (Right inset) Mean displacement $\left\langle d_{i}\right\rangle$ of every monomer ( $x$-axis) and for the different applied forces ( $y$-axis). (Left inset) A section of $\left\langle d_{i}\right\rangle$ as a function of the monomer number $N_{i}$ for three selected values of the forces. The values of $\left\langle d_{i}\right\rangle$ for the forces $F=64$ have been scaled with a factor $\times 10$, and the ones for $F=68$ with a factor $\times 5$.

DNA. In fact, the recombination of the chain after the melting (i.e., for decreasing forces, see lower panels of Fig. 5) takes more time to be achieved, and so the backward paths can be very different from the forward ones, and increase their difference by reducing the $t_{s}$ values.

\section{B. Morse potential with $G \neq 0$}

The inclusion of the barrier makes the overstretching transition sharper, as also seen in the melting temperature studied in Refs. 26 and 27. For this reason, we used here a different value of the overstretching force $\left(F_{o s}=68 \mathrm{pN}\right)$, which gives a better fit in this version of the model. The results of the simulation are drawn in Fig. 6. On the other hand, a higher stability of the chain length after the transition is obtained in this case.

Figure 7 shows an equivalent qualitative behavior of the probability distribution than the $G=0$ case. More interesting is the hysteretic behavior shown in Fig. 8. We

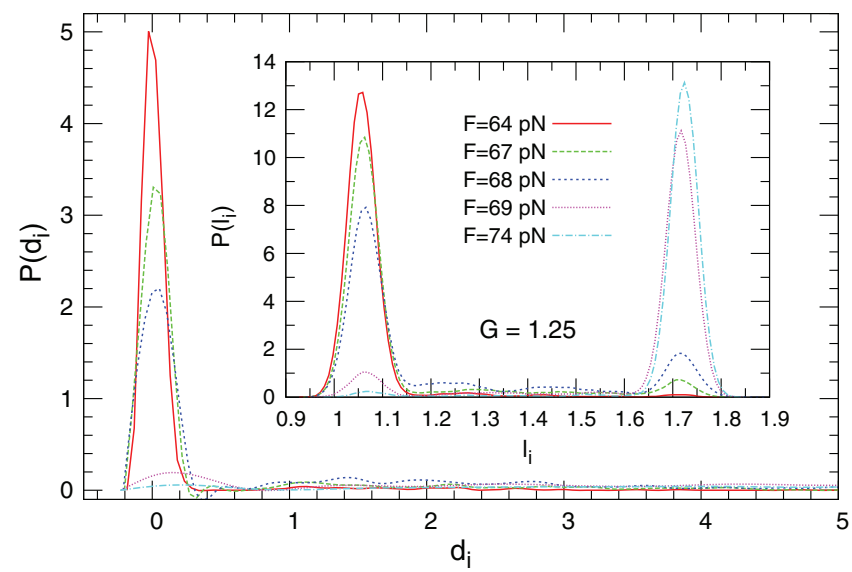

FIG. 7. Probability distribution of the monomer distance $l_{i}$ and of the melting variable $d_{i}$ for different values of the applied force $F$ in the case of $G \neq 0$.
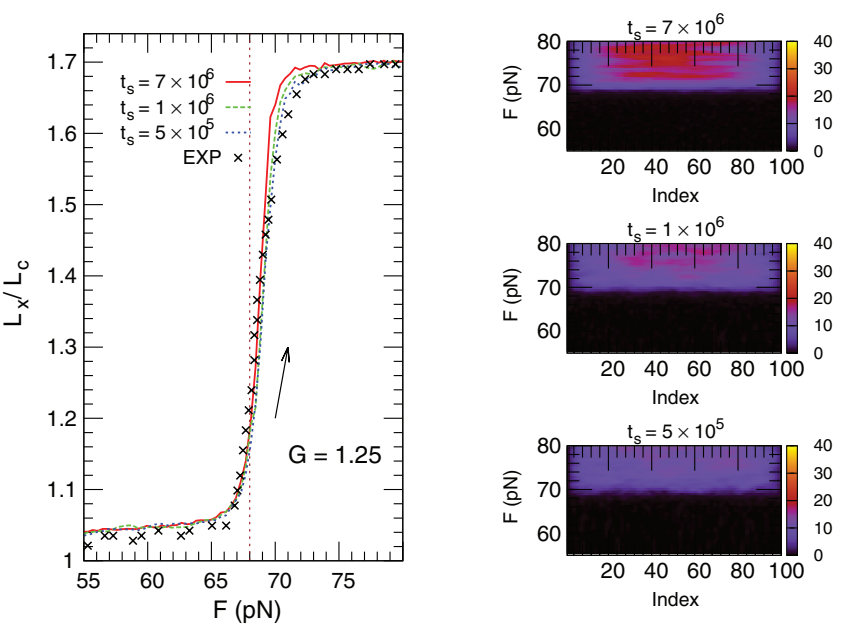

Index
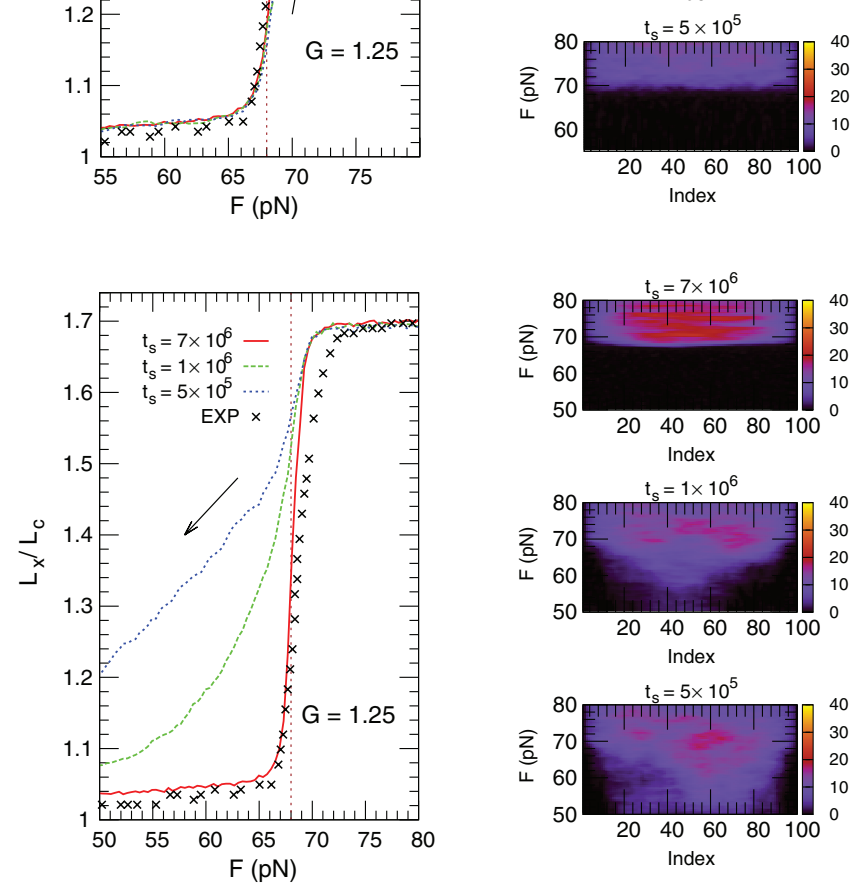

Index

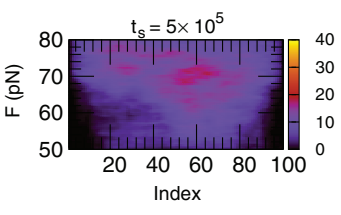

FIG. 8. Transition with different simulation time in the case $G \neq 0$. Upper panel: Forward. Lower panel: Backward.

can see that reducing the simulation times $t_{s}$ the forward curves maintain quite well, and even improve, their agreement with the experimental data (see, for example, the curve with $t_{s}=5 \times 10^{5}$ ), but the backward paths present bigger hysteresis than the simple Morse potential case, which increases by decreasing the values of $t_{s}$. This because the "open" state of the strands is more stable for the presence of the barrier that makes more difficult the recombination of the two single strands of the DNA in a one dsDNA.

\section{The role of the temperature}

One interesting topic in the overstretching transition is the role played by the temperature. Some experimental data have been reported in Ref. 5, where the authors also develop a statistical calculation in order to evaluate a possible analytic law giving the transition force as a function of the temperature. Our model is not able to reproduce the experimental data under the change of $T$. The reason can be due to the presence of the overstretching force $F_{\text {os }}$ as a parameter that control the depth of the Morse potential reported in Eq. (6), where the overstretching force is put by hand in order to give the 


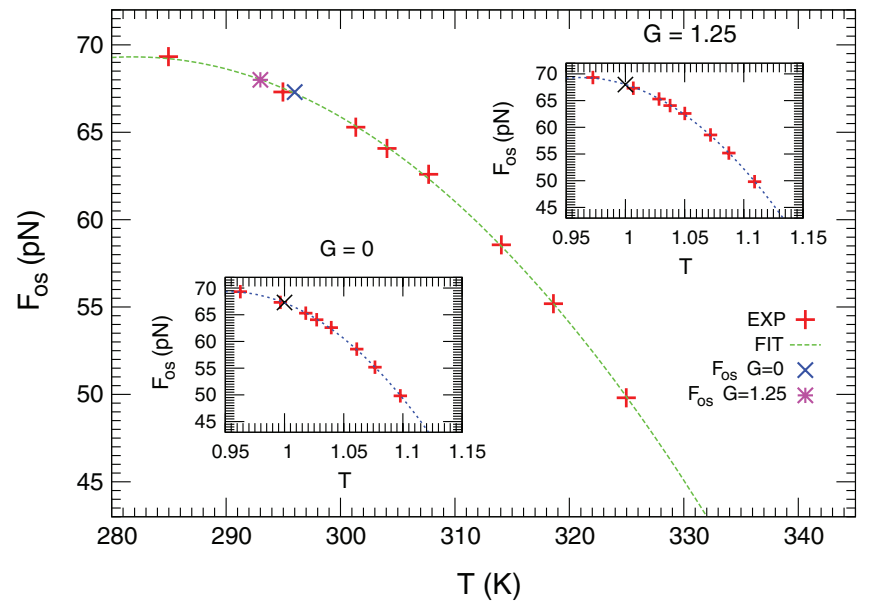

FIG. 9. Experimental data for the dependence of $F_{o s}$ with temperature. The solid line represents a fit curve in all the plots. (Left inset) The scaling of $T$ obtained by using as room temperature the one obtained with $G=0$ (normal Morse potential). (Right inset) The scaling of $T$ obtained in the case of Morse potential with barrier with $G=1.25$ (Morse potential with interaction with the solvent).

experimental curve. For this reason we need to include explicitly the dependence of $F_{o s}$ with temperature. The formula found by Wenner et al. in Ref. 5 seems not to give some physical predictions when extrapolated for low temperatures. For our purpose, and in the range of the temperature we want to test, it is enough to evaluate the dependence of the overstretching force with the temperature just adopting a simple quadratic fit of their experimental data in the form $y=a x^{2}$ $+b x+c$, whose plots are shown in Fig. 9 for the two models (with and without barrier of the Morse potential). We can observe that the fit works very well in this limited range of temperatures. The presence or absence of the barrier gives rise to different scaling of the room temperatures, generated by the different values of the overstretching forces $F_{o s}$ value determined in our model in the two cases. This different values are indicated by the two single points in the plots of the figure.

The calculated curves are reported in Fig. 10 for different temperatures $T$. It is possible to see there that they main-
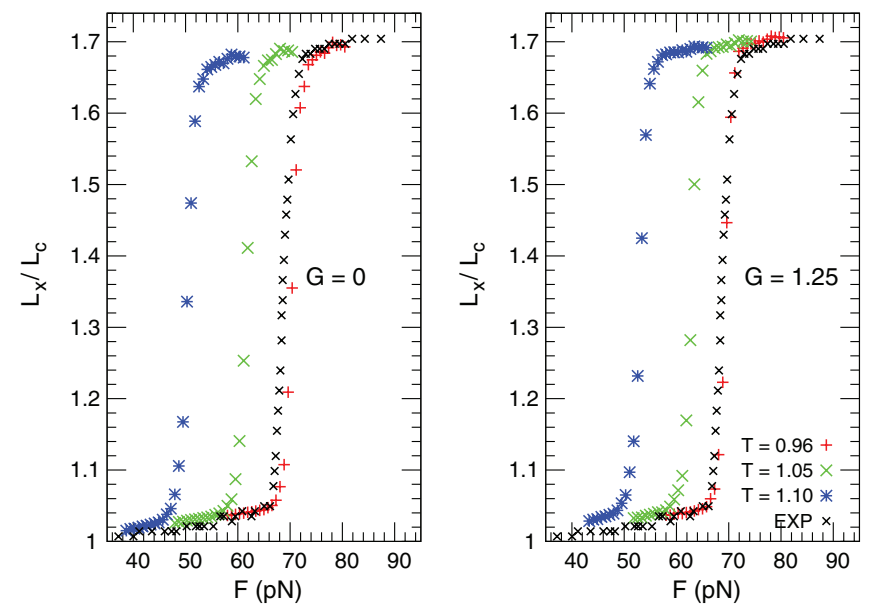

FIG. 10. Stretching at different temperatures with $G=0$ (left panel), and $G \neq 0$ (right panel), by changing $F_{o s}$ with temperature according to the fit of the experimental data.
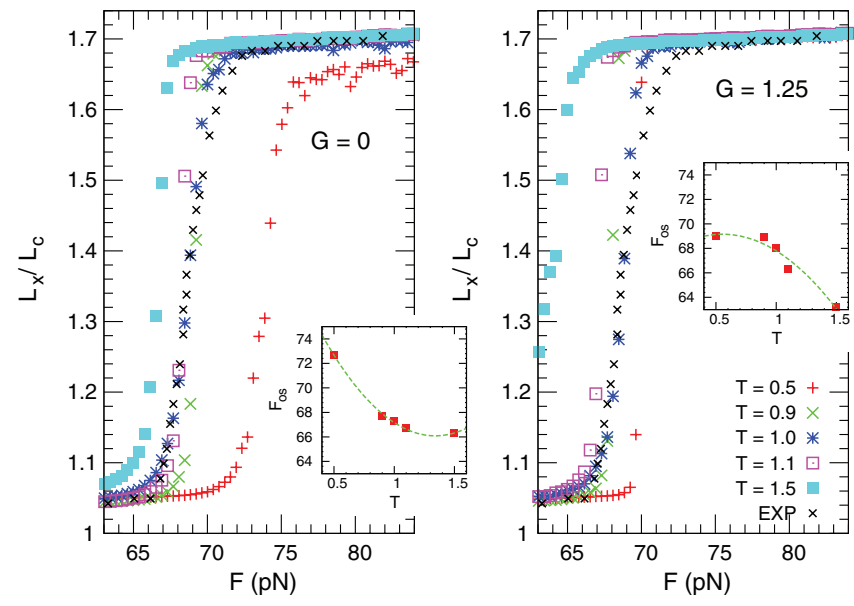

FIG. 11. Stretching at different temperatures where $F_{o s}$ is maintained fixed at the value of the room temperature. Left panel: With $G=0$. Right panel: With $G \neq 0$. (Insets) Values and the evaluated fits of the $F_{o s}$ vs $T$.

tain the same slope shown in Subsections III A-III B. The only difference is that the curves are shifted to recover the given overstretched force. As in Sec. III B, we investigated the effect on the elongation curve in forward and backward experiments, and found again a similar behavior than the one depicted above (not shown), just translated the amount of the overstretching force $F_{o s}(T)$ evaluated at that temperature by means of the fit procedure shown in the insets of Fig. 9 for the two cases $G=0$ and $G \neq 0$.

In these calculation $F_{o s}(T)$ changes as a function of $T$. Conversely, if we maintain fixed the value of $F_{o s}$ in the model for the depth of the potential $D$ as evaluated for the case of room temperature, we find, by changing $T$, the results shown in Fig. 11. The two panels show the $G=0$ and $G=1.25$ cases, respectively. The insets of the two panels show the curves predicted for the overstretching force $F_{o s}$ vs $T$. In both cases they do not reproduce the experimental values reported in Fig. 9. However, it seems important to remark that the two trends are not equivalent. We can notice that the case with $G=1.25$ maintains at least the correct concavity of the $F_{o s}$ vs $T$ dependence, and this would constitute a support toward the possibility that the presence of the maximum in the potential better represents the nucleotide interaction than the standard Morse potential. $^{26,27}$

The explicit insertion of $F_{o s}(T)$ as an experimental parameter in the depth of the Morse potential may represent a limitation of the model. Nevertheless, this limitation is somehow justified by the fact that this picture only takes into account melting properties, detached from other mechanisms that can in principle contribute to the overstretching phenomenon. We argue that a more extended model could take it into account in a proper way.

\section{Anharmonic stacking interaction}

The nonlinear stacking interaction is included in the PBD model to obtain a sharper transition when temperature increases. The stacking contribution represents a cooperative term which is related to the recoil force given by the adjacent 

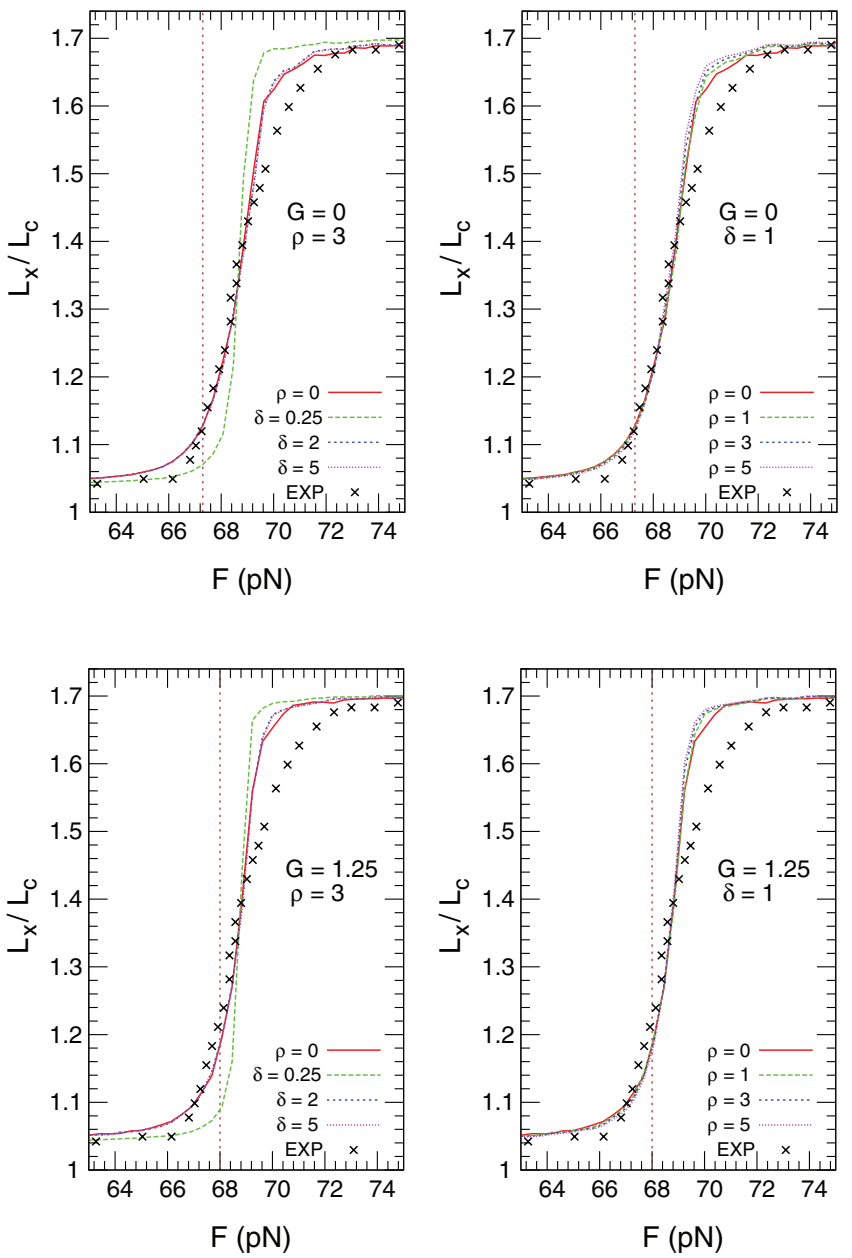

FIG. 12. Dependence of the overstretching transition with the parameters $\rho$ and $\delta$ of the Peyrard cooperative term $W$ in Eq. (5). The panels on the left are calculated with $\rho=3$ and various values of $\delta$. Panels on the right are calculated with $\delta=1$ and different values of $\rho$. Upper panels: Model with $G=0$. Lower panels: Model with $G \neq 0$.

nucleotides of the single DNA strands, which become relevant when the bond with the complementary strand gets weaker, and the adjacent monomers of the same strand tend to avoid the separation of the monomers. The anharmonic contribution (with $\rho \neq 0$ ) increases this effect at short relative distances of the monomers of the same chain, increasing their cooperative effect. Thus, its inclusion, while keeping fixed the rest of the parameter, it is expected to lead to a sharper overstretched transition.

Figure 12 shows the results of the simulations for both the cases $G=0$ and $G \neq 0$, confirming that expectation. The curves plotted are calculated with different values of the parameters $\delta$, giving the mean distance of that amplification, and $\rho$, giving the corresponding amplitude.

In both the potentials large values of parameter $\rho$ and small values of $\delta$ make the transition sharper, similar to the melting transition with temperature. Our ansatz is that these parameters, as well as the parameters of the Morse potential, can be associated with experimental conditions as $\mathrm{pH}$, salt concentration, etc., that has been probed to affect overstretching transition.

\section{CONCLUDING REMARKS}

DNA overstretching transition is one of the most intriguing phenomena among the mechanical properties of this molecule. It is hard to properly model the full interactions that could influence the transition. Then, it makes sense to adopt a minimalist approach to the problem by analyzing only those interactions that hopefully contribute to the phenomenon. In this paper, we have focused on the effect of force induced melting in the overstretching transition. For that we have used the well known PBD model which relates, in a very simple way, both mechanical and thermal properties of DNA. ${ }^{26}$ In order to couple the melting degrees of freedom with the stretched polymer we have proposed a phenomenological dependence of PBD parameter on the external force. In this sense, by using the equations Eqs. (1)-(5), we are able to fairly reproduce the basic experimental outcomes. In particular, the overstretching transition is reproduced with a reasonable approximation. The basic idea of this work is to join melting variables with the mechanical deformation of the chain, following that way the commonly recognized idea that consider melting as responsible of the DNA overstretching transition. Although the model oversimplifies the DNA structure, it still contains the main ingredients of the molecule dynamics, especially the phenomena related to non-equilibrium properties. For instance, an important feature of the model is the ability to reproduce the hysteretic behavior of the transition. In our model this is a purely dynamical effect due to the differences in the relaxation times between the pulling and the releasing force application. This fact is enhanced by the presence of the barrier in the modified PBD model, which takes into account solvent interaction. ${ }^{26,27}$ For this reason, we can conjecture that the presence of a barrier in the base interaction can constitute a more realistic ingredient in the description of the DNA melting/stretching phenomenon. Beside of presenting clear advantages, as the reduced number of variables by using a coarse-grained description and the usage of simple dynamical equations, the model presents some limitations, i.e., the bad reproduction of the behavior of overstretching force with temperature without its explicit introduction in the model as an additional parameter. However, the model can be easily extended to include other aspects not present in this description, such as torsion or a more microscopic picture, that hopefully could account for this kind of behaviors.

\section{ACKNOWLEDGMENTS}

This work is supported by the Spanish DGICYT Projects No. FIS2011-25167, co-financed by FEDER funds, and by the Comunidad de Aragón through a grant to the FENOL group.

${ }^{1}$ C. Bustamante, Z. Bryant, and S. B. Smith, Nature (London) 421, 423 (2003)

${ }^{2}$ S. B. Smith, Y. Cui, and C. Bustamante, Science 271, 795 (1996).

${ }^{3}$ P. Cluzel, A. Lebrun, C. Heller, R. Lavery, J. L. Viovy, D. Chatenay, and F. Caron, Science 271, 792 (1996).

${ }^{4}$ M. C. Williams, J. R. Wenner, I. Rouzina, and V. A. Bloomfield, Biophys. J. 80, 874 (2001).

${ }^{5}$ J. R. Wenner, M. C. Williams, I. Rouzina, and V. A. Bloomfield, Biophys. J. 82, 3160 (2002).

${ }^{6}$ J. vanMameren et al., Proc. Natl. Acad. Sci. U.S.A. 106, 18231 (2009). 
${ }^{7}$ J. F. Léger, G. Romano, A. Sarkar, J. Robert, L. Bourdieu, D. Chatenay, and J. F. Marko, Phys. Rev. Lett. 83, 1066 (1999).

${ }^{8}$ C. Storm and P. Nelson, Europhys. Lett. 62, 760 (2003).

${ }^{9}$ S. Cocco, J. Yan, J.-F. Leger, D. Chatenay, and J. F. Marko, Phys. Rev. E 70, 011910 (2004)

${ }^{10} \mathrm{H}$. Fu, Hu Chen, J. F. Marko, and J. Yan, Nucleic Acids Res. 38, 5594 (2010).

${ }^{11}$ X. Zhang, H. Chen, H. Fu, P. S. Doyle, and J. Yan, Proc. Natl. Acad. Sci. U.S.A. 109, 8103 (2012).

${ }^{12}$ P. Gross et al., Nat. Phys. 7, 731 (2011)

${ }^{13}$ D. Paik and T. Perkins, J. Am. Chem. Soc. 133, 3219 (2011).

${ }^{14}$ N. Bosaeus, A. H. El-Sagheer, T. Brown, S. B. Smith, B. kerman, C. Bustamante, and B. Nordn, Proc. Natl. Acad. Sci. U.S.A. 109, 15179 (2012).

${ }^{15}$ G. A. King, P. Gross, U. Bockelmann, M. Modesti, G. J. L. Wuite, and E. J. G. Peterman, Proc. Natl. Acad. Sci. U.S.A. 110, 3859 (2013).

${ }^{16}$ X. Zhang, H. Chen, S. Le, I. Rouzina, P. S. Doyle, and J. Yan, Proc. Natl. Acad. Sci. U.S.A. 110, 3865 (2013).

${ }^{17}$ P. Cizeau and J. L. Viovy, Biopolymers 42, 383 (1997).

${ }^{18}$ J. F. Marko, Phys. Rev. E 57, 2134 (1998)

${ }^{19}$ M. Santosh and P. Maiti, J. Phys. Condens. Matter 21, 034113 (2009).
${ }^{20}$ A. Hanke, M. G. Ochoa, and R. Metzler, Phys. Rev. Lett. 100, 018106 (2008).

${ }^{21}$ F. Romano, D. Chakraborty, J. Doye, T. E. Ouldridge, and A. A. Louis, J. Chem. Phys. 138, 085101 (2013)

${ }^{22}$ A. Fiasconaro and F. Falo, Phys. Rev. E 86, 032902 (2012).

${ }^{23}$ F. Manca, S. Giordano, P. L. Palla, F. Cleri, and L. Colombo, Phys. Rev. E 87, 032705 (2013).

${ }^{24}$ R. W. Ogden, G. Saccomandi, and I. Sgura, J. Nonlinear Math. Phys. 18(suppl.2), 411 (2011).

${ }^{25}$ T. Dauxois, M. Peyrard, and A. R. Bishop, Phys. Rev. E 47, 684 (1993).

${ }^{26}$ G. Weber, Europhys. Lett. 73, 806 (2006).

${ }^{27}$ R. Tapia-Rojo, J. J. Mazo, and F. Falo, Phys. Rev. E 82, 031916 (2010).

${ }^{28}$ S. Cuesta-Lopez, J. Errami, F. Falo, and M. Peyrard, J. Biol. Phys. 31, 273 (2005).

${ }^{29}$ N. K. Voulgarakis, A. Redondo, A. R. Bishop, and K. O. Rasmussen, Phys. Rev. Lett. 96, 248101 (2006).

${ }^{30}$ A. Singh, B. Mittal, and N. Singh, Phys. Express 3, 18 (2013).

${ }^{31}$ G. Weber, J. W. Essex, and C. Neylon, Nat. Phys. 5, 769 (2009).

${ }^{32}$ P. Bianco, L. Bongini, L. Melli, M. Dolfi, and V. Lombardi, Biophys. J. 101, 866 (2011)

${ }^{33}$ S. Whitelam, S. Pronk, and P. L. Geissler, Biophys. J. 94, 2452 (2008). 